Supplementary Information

\title{
Portable, Constriction-Expansion Blood Plasma Separation and Polymerization-Based Malaria Detection
}

\author{
Tatyana A. Shatova ${ }^{1}$, Shefali Lathwal ${ }^{1}$, Marissa R. Engle ${ }^{1}$, Hadley D. Sikes ${ }^{1 *}$, Klavs F. Jensen ${ }^{1 *}$
}

${ }^{1}$ Department of Chemical Engineering, Massachusetts Institute of Technology, Cambridge, MA 02139

* Corresponding Authors:

Hadley D. Sikes: Email: sikes@mit.edu Phone: 617.253.5224 Fax: 617.253.2272.

Klavs F. Jensen: Email: kfjensen@mit.edu Phone: 617.253.4589 Fax: 617.253.6956

\section{Table of Contents}

Figure S-1 Visual comparison of 100 and 2,800 isolate separation ratios for whole blood................S-2

Figure S-2 Effect of isolate separation ratio on background color of paper assays.....................

Table S-1 Red blood cell lysis comparison for decanting, centrifuging, and device processing........... S-4

Figure S-3 Colorimetric intensity vs. Plasmodium falciparum histidine-rich protein 2 concentration.... S-5

Table S-2 Statistical analysis of color intensity vs. background S-6 
Figure S-1: Visual comparison of 100 isolate separation ratio (or 99\% purity previously shown in the literature), 2,800 isolate separation ratio (or $99.96 \%$ purity achieved by the device described in this manuscript), and pure plasma. The background is grayed out in order to emphasize the color difference for a twenty-eight-fold improvement in separation capability using the $5^{\circ}$ expansion design.

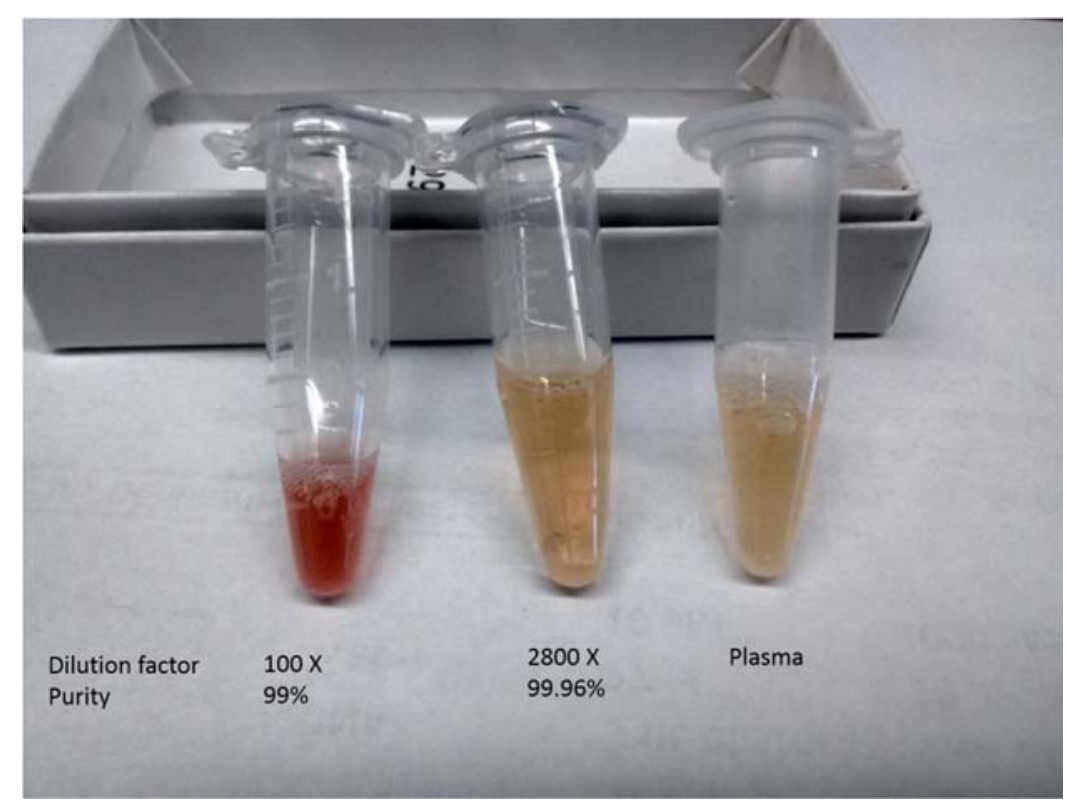


Figure S-2: Effect of purity of plasma sample on background color of paper tests. $10 \mu \mathrm{L}$ samples of pure plasma (prepared via centrifugation), 100 isolate separation ratio (or 99\% purity previously shown in the literature), and 2,800 isolate separation ratio (or $99.96 \%$ purity achieved by the device described in this manuscript) were applied to paper surfaces for one hour in a humid chamber. (a) Representative images of paper surfaces containing $10 \mu \mathrm{L}$ sample (Wet), blotted sample without washing ( 0 Wash), with one wash of $20 \mathrm{uL}$ solution of $1 \mathrm{X}$ PBS (1 Wash), and two washes each of $20 \mu \mathrm{L}$ solution of 1X PBS (2 Wash) are shown. (b) Absolute difference in the mean colorimetric intensity of paper surfaces incubated with $99 \%$ and $99.96 \%$ pure plasma samples compared to surfaces incubated with pure plasma. The error bars represent standard deviation $(n=6)$. The plasma samples with $99.96 \%$ purity are visually and quantitatively similar to the pure plasma samples and a single buffer wash is sufficient to remove any background color from these surfaces. The samples with $99 \%$ purity produce a visible red hue on the paper surface even after two washes; this background color can lower the visual contrast and interfere with accurate interpretation of positive test results.

(a)

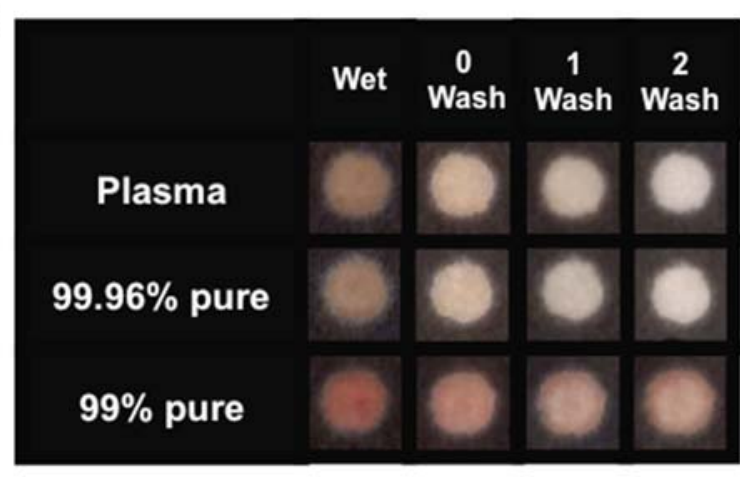

(b)

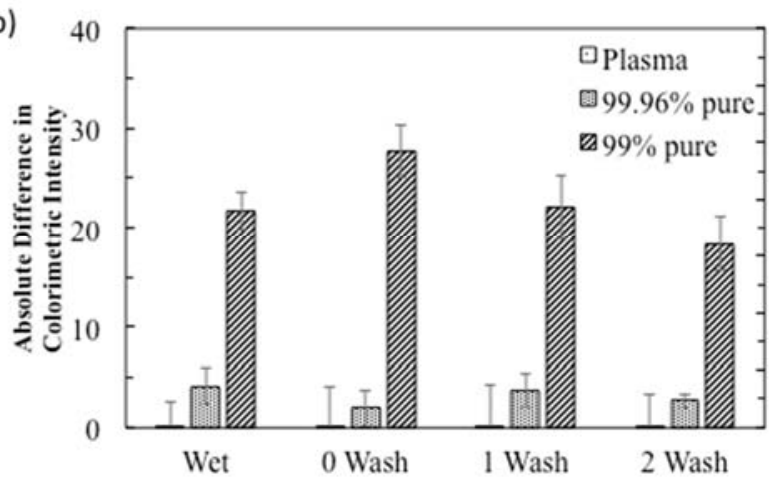


Table S-1: Mean percent lysis for red blood cells using three different methods for second day old undiluted whole blood. Decanting separation involved direct plasma removal (using a pipettor) from a BD Vacutainer tube (Becton Dickinson) that was stored overnight in a refrigerator in order to allow for cell settling and plasma decanting. Centrifuge separation (laboratory standard) involved $1.5 \mathrm{~mL}$ Eppendorf LoBind tubes (Sigma Aldrich) that were spun down at $1 \mathrm{~g}$ for $10 \mathrm{~min}$ before plasma was removed from the top using a pipette. Device separation was done for undiluted blood on a $5^{\circ}$ expansion device for $50-300 \mu \mathrm{L} / \mathrm{min}$ flow rates, which led to similar low lysing values. The \% red blood cell lysed was calculated using the Cripps Method. ${ }^{1-3}$

$\begin{array}{ccc}\text { Method } & \text { Ave \% Lysis } & \text { 1 Std Dev of \% Lysis } \\ \text { Decanting } & 0.059 \% & 0.006 \% \\ \text { Centrifuge } & 0.095 \% & 0.04 \% \\ \text { Device } & 0.11 \% & 0.02 \%\end{array}$


Figure S-3: Mean colorimetric intensity values with background subtracted, in which concentration of Plasmodium falciparum histidine-rich protein 2 (PfHRP2) was varied in undiluted whole blood. +/- 1 standard deviation is calculated using a pooled variance approach. The details of quantification have been published before. ${ }^{4}$

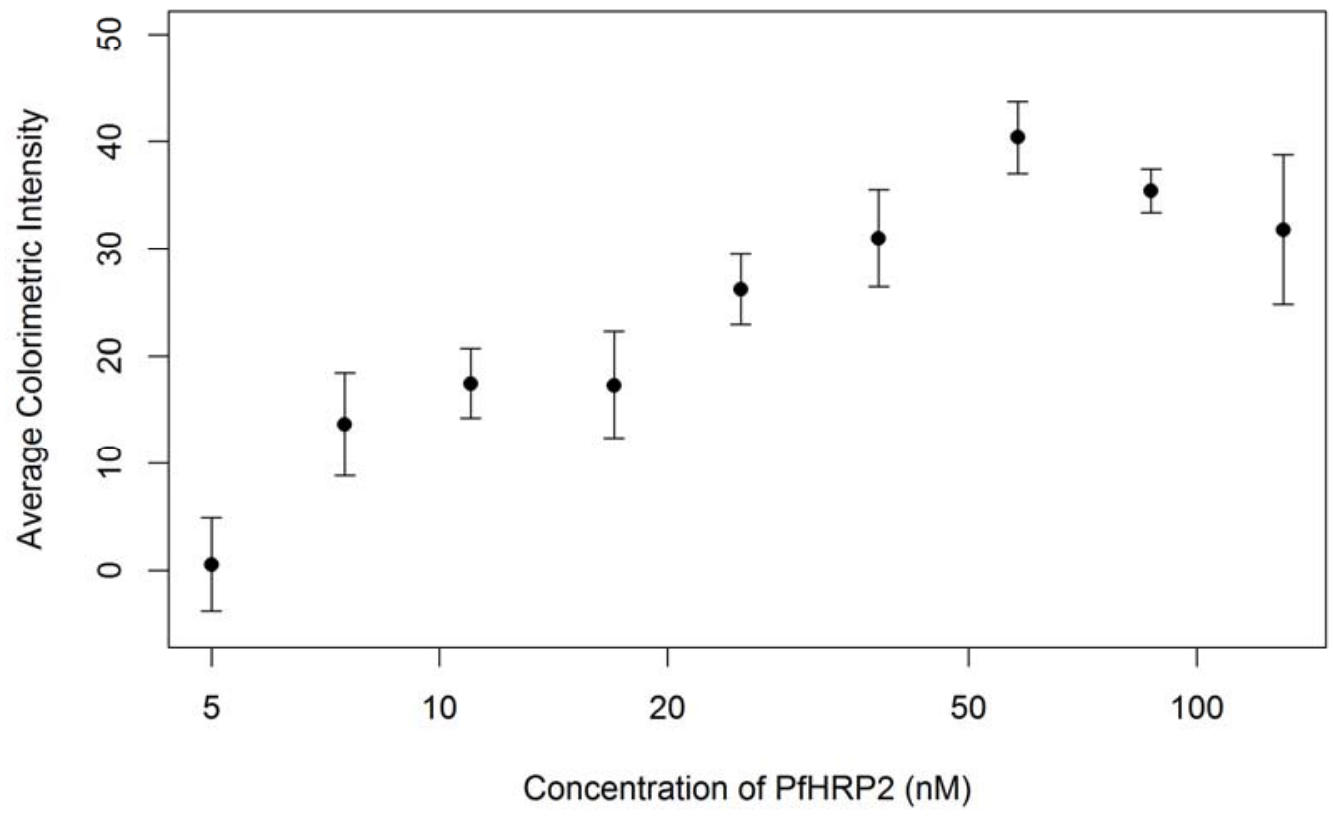


Table S-2: Concentration of PfHRP2 vs. p-value for the 1-tail unpaired t-test. P-value of less than 0.05 shows statistically significant difference between color intensity and background. Concentration of $7.5 \mathrm{nM}$ and above was found to be statistically above the background value.

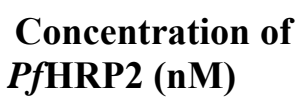

0$$
5
$$$$
7.5
$$

11

17

25

38

58

87

130

Average
Colorimetric
Intensity

13.60

14.14

27.24

31.04

30.91

39.78

44.57

53.97

49.02

45.39

Std. Dev. of
Colorimetric
Intensity

2.02

7.26

8.09

5.18

8.32

5.33

7.62

5.37

2.91

11.96
P-value

vs.

Background

0.4554

0.0461

0.0088

0.0309

0.0036

0.0073

0.0012

0.0001

0.0204

\section{References:}

(1) Cripps, C. M. Journal of Clinical Pathology 1968, 21, 110-112.

(2) Malinauskas, R. A. Artificial Organs 1997, 21, 1255-1267.

(3) Han, V.; Serrano, K.; Devine, D. V. Vox Sanguinis 2010, 98, 116-123.

(4) Badu-Tawiah, A. K.; Lathwal, S.; Kaastrup, K.; Al-Sayah, M.; Christodouleas, D. C.; Smith, B. S.; Whitesides, G. M.; Sikes, H. D. Lab on a Chip 2015, 15, 655-659. 